\title{
CAPÍTULO 37: AVALIAÇÃO DO DESEMPENHO DO GESTOR NAS COMPETÊNCIAS GERENCIẢIS EM UMA UNIDADE DE ALIMENTAÇÃO E NUTRIÇÃO
}

\section{CHAPTER 37: EVALUATION OF THE MANAGER'S PERFORMANCE IN THE MANAGEMENT SKILLS IN A FOOD AND NUTRITION UNIT}

\author{
Maria Rosa Figueiredo Nascimento ${ }^{1}$; Alexandra Marins Hatschek ${ }^{2}$; Beatriz de Lopes ${ }^{3}$; Katia \\ Cansanção Correa de Oliveira ${ }^{4}$; Vânia Madeira Policarpo ${ }^{5}$
}

\begin{abstract}
Resumo
A presença de um gestor é fundamental para garantir a qualidade do serviço em uma Unidade de Alimentação e Nutrição (UAN). Nesse sentido, o objetivo do presente estudo foi caracterizar um gestor de UAN, bem como, suas atividades dentro desse serviço, e também identificar percepções a respeito das competências gerenciais requeridas pela empresa e as efetivas em seu trabalho enquanto gestor. Trata-se de um estudo de campo, descritiva, realizado em uma Unidade de Alimentação e Nutrição de escola municipal entre março a junho de 2018. As informações de interesse, foram obtidas por meio de observações e perguntas abertas conduzidas por uma aluna de pós-graduação. A escola atende 711 pessoas entre alunos do primeiro e segundo seguimento do ensino fundamental e funcionários. Inicialmente, na ausência de um gestor foi observado que os colaboradores executavam as suas atribuições de forma irregular não seguiam nenhuma sequência, consequentemente o serviço tornava se desorganizado e cansativo. Após a chegada de uma gestora na UAN, houve capacitação dos funcionários, implementação de procedimentos operacionais padronizados (pops) e de fichas técnicas de preparos, além de melhora na comunicação entre a equipe, refletindo na qualidade do serviço. Podemos concluir que, a presença do gestor nessa UAN foi fundamental para instituir e padronizar técnica que visam obter qualidade ponto de vista higiênico-sanitário, desde o início da produção até o produto final, assim como para melhorar a interação entre a equipe.
\end{abstract}

Palavras-Chave: Gestão, Unidade de alimentação e nutrição, Motivação, Conscientização.

\begin{abstract}
The presence of a manager is essential to guarantee the quality of service in a Food and Nutrition Unit (UAN). In this sense, the objective of the present study was to characterize a UAN manager, as well as his activities within that service, and also to identify perceptions regarding the managerial competencies required by the company and the effective ones in his work as a manager. This is a descriptive field study carried out in a Food and Nutrition Unit of a municipal school between March and June 2018. The information of interest was obtained through observations and open questions conducted by a graduate student. The school serves 711 people, including students in the first and second segments of elementary school and employees. Initially, in the absence of a manager, it was observed that employees performed their assignments irregularly and did not follow any sequence, consequently the service became disorganized and tiring. After the arrival of a manager at the UAN, there was training for employees, implementation of standardized operational procedures (pops) and technical preparation sheets, as well as improved communication between the team, reflecting

\footnotetext{
${ }^{1}$ Doutora em Ciência e Tecnologia de Alimentos, PPGCTA/UFRRJ, mariarosa@ufrrj.br

${ }^{2}$ Discente do curso de pós-graduação lato sensu núcleo de pós-graduação e extensão - Faveni

${ }^{3}$ Mestranda em Ciência e Tecnologia de Alimentos pela (PPGCTA / UFRRJ)

${ }^{4}$ Doutoranda em Ciências Nutricionais, Instituto de Nutrição Josué de Castro UFRJ, kcansancao@gmail.com

${ }^{5}$ Mestre em Ciência e Tecnologia de Alimentos, PPGCTA / UFRRJ, madeira@ufrrj.br
} 
on the quality of the service. We can conclude that the presence of the manager in this UAN was fundamental to institute and standardize techniques that aim to obtain quality from a hygienic-sanitary point of view, from the beginning of production to the final product, as well as to improve the interaction between the team.

Keywords: Management, Food and nutrition unit, Motivation, Awareness.

\section{Introdução}

Atualmente encontramos muitos casos de produção onde a rentabilidade dos funcionários está em nível decrescente por consequência da falta de motivação ou da falta de sincronia no meio de trabalho (OLIVEIRA, FERREIRA, TEIXEIRA, 2020; JUSTEN, CHILANTI, 2018). É importante citar que, o setor de serviço de alimentação não se faz de forma individual, pelo contrário, existe no contexto um setor de redes humanas, que desencadeiam as atividades dos funcionários na maioria do tempo, de forma que equipe e gerência se interrelacionam, favorecendo a produtividade dos setores de serviço, ou seja, a motivação de funcionários numa Unidade de Alimentação e Nutrição (UAN) atua como um fator de melhoria no desempenho dos profissionais (SANTOS, BUCCIOLI, 2019; AVELATO, ARAUJO, 2009; BITENCOURT, 2005; TEIXEIRA, 2010).

Para se obter qualidade, a UAN deve contar com um quadro de pessoal adequado tanto do ponto de vista quantitativo como qualitativo, desenvolvendo ações proativas, antecipandose nas suas ações, prevenindo desvios comportamentais e de desempenho profissional que possam prejudicar a qualidade dos serviços e produtos (AMARAL, CARNEVALE, 2008). As UANs são formadas por um conjunto de áreas e serviços responsáveis pela produção de refeições balanceadas dentro dos padrões dietéticos e higiênicos, visando atender as necessidades nutricionais de seus comensais, adequadas aos recursos financeiros da unidade (DE BRITO, TORRES, 2015; ABREU et al., 2013).

Com o forte crescimento do setor de serviços, a grande concorrência, a presença de doenças transmitidas por alimentos e processos devido à baixa qualidade de produtos, a qualidade de produtos e serviços passou a ser um dos objetivos a serem alcançados pelas UANs, a fim de promover a saúde de seus usuários e garantir o seu lugar no mercado de alimentação (ALVAREZ, 2019; SLACK, 2018; TEIXEIRA et al., 2010). Desse modo, o êxito no funcionamento de uma UAN, depende da definição clara de seus objetivos, de sua estrutura administrativa, de suas instalações físicas e recursos humanos e, sobretudo da normatização de todas as operações desenvolvidas, que devem ser baseadas nos elementos do processo administrativo básico (TEIXEIRA et al., 2010).

Segundo Abreu (2013), o sucesso de uma organização é baseado nos processos pelo qual a mesma opera, podendo ser o processo administrativo o responsável. Este processo, 
pode ser definido como as várias funções do administrador de UAN como um todo. $\mathrm{O}$ planejamento, organização, direção e controle, constituem as funções administrativas. Quando visualizadas na sua abordagem total para o alcance de objetivos, tais como, a qualidade de um serviço, forma o processo administrativo. A qualidade pode ser conceituada como uma sistematização da produção de produtos e serviços com o intuito de assegurar um resultado econômico das operações, e que atendam plenamente a satisfação do consumidor, o conceito de qualidade é uma ideia antiga, porém sua gestão para obtenção é recente (ABREU et al., 2013; COLARES, FREITAS, 2005). Bravo (2010) descreve que, a gestão de qualidade facilita o processo de mudanças organizacionais envolvendo pessoas e criando processos que facilitem o alcance da mudança esperada.

O engenheiro francês Henry Fayol, é considerado um dos fundadores da administração moderna. Fayol dedicou sua vida ao estudo dessa área, e desenvolveu a teoria da gestão ou processo administrativo, onde segundo ele, "administração é baseada em quatro princípios (planejamento, organização, direção e controle), de acordo com o autor, previsão seguido de métodos adequados de gerência torna os resultados satisfatórios inevitáveis, afirma ainda que esses princípios são universais e podem ser aplicados a qualquer organização" (FAYOL, 1994).

Em se tratando de cargos gerenciais, estes exercem um papel de responsabilidade quanto ao sucesso organizacional, uma vez que são os articuladores das pessoas e resultados, atuando como canal de comunicação mediante a coordenação das atividades de suas unidades com as demais unidades de uma organização (SARTOR, ALVES, 2019; FREITAS, ODELIUS, 2018). Uma das áreas onde a atuação do nutricionista brasileiro tem sido frequentemente requerida é a de produção de refeições para atendimento ao trabalhador da indústria, do comércio e do setor de serviços (CFN, 2015; BOOG et al., 1988).

"Se tratando de uma cozinha, trabalha-se com pessoas e para pessoas. É importante observar suas expectativas, entender suas motivações e necessidades, e fazer com que seus anseios se transformem em uma experiência positiva" (MAXIMIANO, 2011).

"A cordialidade, o respeito, o profissionalismo e a eficiência são fatores fundamentais na relação entre gestores e equipe" (FONSECA, 2018).

Os três grandes pilares do serviço de alimentação são: em primeiro lugar o ambiente não só em relação aos aspectos decorativos, mas o ambiente gerado por todo o conjunto humano e físico; em segundo lugar o serviço deve ser adequado ao conceito do restaurante e sua proposta de atendimento ao nicho específico de mercado; e por último, o cardápio que precisa estar em 
perfeita sintonia com todos os elementos componentes desse conceito e em sincronia com os anseios e expectativas do público-alvo (MANZALLI, 2010; ABREU et al., 2013).

Desse modo, para garantir a qualidade do serviço em uma UAN, é fundamental a presença de um gestor visando planejar, organizar, e administrar corretamente toda operação, alcançando menor desperdício de tempo e recursos, aproveitando melhor a mão de obra, reduzindo custos e evitando problemas que ponham em risco a saúde do trabalhador e de seus comensais (NOBREGA et al., 2012; SILVA, BRASIL, 2012). Sendo assim, pesquisas que retratam a importância do gestor dentro de UAN para garantir o controle higiênico-sanitário, do início até o final da produção são fundamentais.

O objetivo do presente estudo foi caracterizar um gestor de UAN, bem como, suas atividades dentro desse serviço, e também identificar percepções a respeito das competências gerenciais requeridas pela empresa e as efetivas em seu trabalho enquanto gestor.

\section{Material e Métodos}

Trata-se de um estudo qualitativo, de metodologia exploratória, que utilizou observações e perguntas abertas, direcionadas à funcionários de uma UAN de uma escola municipal, localizada em Seropédica, Rio de Janeiro. Essa escola, conta com a parceria da Universidade Federal Rural do Rio de Janeiro (UFRRJ), o que à diferencia das demais do mesmo município.

Para obter as informações pertinentes ao estudo, foram realizadas visitações, observações e anotações, visando caracterizar o funcionamento da escola, o número total de funcionários e de alunos, levantamento de mudanças na cozinha, desempenho das atividades desenvolvidas na cozinha pelos funcionários antes e após a presença de um gestor nessa UAN

As visitas ocorreram entre os meses de março a junho de 2018 nos horários de 07:00 às 14:00 horas. Adicionalmente, foram realizadas entrevistas abertas, ou seja, os participantes foram orientados a falar livremente a respeito da temática proposta, possibilitando ao pesquisador um amplo discurso acerca do tema (BONI, QUARESMA, 2005). Tanto as visitas quanto as entrevistas foram conduzidas pela pós-graduanda. Cabe ressaltar que, as observações realizadas contribuíram para complementar as informações obtidas, por meio da percepção de aspectos comportamentais (MINAYO, 2010). 


\section{Resultados e Discussão}

Quanto a caracterização da escola, foi constatado que é uma escola municipal, mas tem parceria com a Universidade Federal Rural do Rio de Janeiro, sendo administrada por uma diretora da universidade e uma subdiretora da prefeitura. Observou-se que a gestão e democrática, partindo da premissa de que todos são importantes, por isso devem ser ouvidos e respeitados. É um modelo de trabalho mais colaborativo, que depende do empenho conjunto para alcançar ótimos resultados. Nesse caso, o mais comum é ouvir frases do tipo "não se gerencia pessoas, se gerencia com pessoas". É um tipo mais equilibrado no qual todos colaboram para chegar a um denominador comum. O difícil é achar o ponto de equilíbrio ideal para o empreendimento.

A escola atende ao primeiro e segundo seguimento do ensino fundamental, sendo 350 do primeiro e 240 do segundo seguimento, 53 professores, 48 funcionários sendo que, 8 pertence a universidade e 21 a prefeitura Municipal de Seropédica, também conta com a ajuda dos terceirizados, sendo 15 pagos pela universidade e 4 pela prefeitura, totalizando 691 pessoas. Adicionalmente, há colaboração de 20 estagiários distribuídos em todos os setores da escola (Tabela 1).

Tabela 1. Caracterização da escola.

\begin{tabular}{cc}
\hline Função & Número de pessoas \\
\hline Alunos do $1^{\circ}$ segmento & 350 \\
Alunos do $2^{\circ}$ segmento & 240 \\
Professores & 53 \\
Outras funções & 48 \\
Estagiários & 20 \\
\hline Total & 711 \\
\hline
\end{tabular}

Observou se que a cozinha tem dois funcionários da universidade, 01 cozinheiro e 01 técnico, além de 08 merendeiras do quadro da prefeitura. A cozinha funciona em horário integral (das 07:00 às 17:00 horas), servindo desjejum, almoço e lanche, o que destaca essa escola das demais do mesmo município, pois é a única que atende horário integral. Durante a permanência do estágio foi visto que não havia nenhum profissional que gerenciasse, comandasse e fiscaliza se as atividades desenvolvidas na cozinha, na qual foi observado que, os colaboradores executavam as suas atribuições de forma irregular não seguiam nenhuma sequência, consequentemente o serviço tornava se desorganizado e cansativo. Para Teixeira et al. (2010), o êxito no funcionamento de uma unidade de alimentação e nutrição, depende da definição clara de seus objetivos, de sua estrutura administrativa, de suas instalações físicas e recursos humanos e, sobretudo da normatização de todas as operações desenvolvidas, que 
devem ser baseadas nos elementos do processo administrativo básico, preconizado por Fayol.

Quanto a execução do cardápio, os mesmo já elaborados pela prefeitura, observou se que os colaboradores executavam de qualquer maneira, não aplicando as técnicas de prépreparo, preparo e cocção, as Boas Práticas de Fabricação (BPFs) de acordo com a RDC 216 de 2004, não eram aplicadas tão pouco os procedimentos operacionais padronizados (POPs) (BRASIL, 2004). Segundo Paladini (2012), as estratégias de produção da qualidade iniciamse, sem dúvida, pela introdução de processos de gestão de qualidade, a ideia de tais estratégias é relativamente simples. Trata-se de um processo que visa induzir as pessoas a produzir qualidade em suas atividades desde o início. Para Mezomo (2014), os nutricionistas administradores do serviço de nutrição devem estabelecer sistemas de gestão com uma estruturação própria, de acordo com os objetivos preestabelecidos, a fim de alcançar tais objetivos e minimizar possíveis problemas.

Quanto ao comprimento do horário de entrada e saída dos colaboradores foi visto que eles faltavam com frequência, chegavam atrasados e às vezes saiam mais cedo, com essa falta de compromisso com o serviço muitas vezes as refeições saiam mais tarde, atrasando todo serviço. Todos os dias ocorriam algum incidente na cozinha, prejudicando todo o andamento da mesma. De acordo com Abreu (2013), é fundamental que todos os funcionários entendam a importância de cada atividade desenvolvida dentro de uma UAN, e suas atribuições especificas assim como seus horários, a fim de garantir uma rotina saudável e adequada tanto para o bom funcionamento do serviço, quanto para a qualidade de saúde de cada funcionário.

Desse modo, a diretora solicitou ao reitor da universidade parceira, um profissional técnico para administra a UAN. Com a vinda do gestor, houve mudanças drásticas na cozinha, uma gestora que realmente soube administrar, mas também foi um líder. Seu primeiro contato foi uma conversa informal colocando tudo o que ela pretendia fazer ali junto aos colaboradores. Percebeu-se, mudanças na cozinha quanto a instalação de equipamentos em desuso existentes que se encontravam num depósito, com a implantação desse equipamento facilitou os serviços, e foi claramente percebido que os colaboradores desenvolviam suas atividades com prazer, e também o bom relacionamento com a gestora, e a cada dia que passava sentia um grande progresso na cozinha.

Durante o período de férias escolares, foram ministrados cursos de capacitação para os manipuladores como, técnicas de preparo dos alimentos, boas práticas de higiene e de procedimentos operacionais padronizados com o objetivo de melhorar a qualificação dos profissionais promovendo melhora no funcionamento da cozinha. Após a capacitação, foram feitos os POPs e cartazes que foram colocados na cozinha. Como exemplo, os POPs da 
higienização das mãos, da lavagem e desinfecção dos legumes, e cartazes com informações dos horários de almoço dos funcionários da cozinha, técnicas de preparo com as fichas técnicas, introdução de algumas preparações com verduras, hortaliças e frutas no cardápio. Os POPs são ferramenta fundamentais para auxiliar a obtenção de níveis de segurança alimentar previstos na legislação (MOREIRA, DAL FABBRO, 2018; ROSA, GUELBERT, 2105).

Quantos as preparações os colaboradores receberam instruções de como lidar com as fichas técnicas, observando que as preparações passaram a ter gosto, com uma ótima aparência e textura, e diminuição de desperdícios, e melhor aceitabilidade. Adicionalmente, a higienização da cozinha feita diariamente, apresentou melhora, sendo observado que todo final de semana e feito uma limpeza geral. Além disso, o uso de uniforme passou a ser obrigatório. A utilização de ferramentas como as fichas técnicas, treinamentos e diálogo, são fundamentais para garantir a qualidade do serviço, a importância de cada etapa e das obrigatoriedades dentro de uma UAN precisam ser claras de forma que o colaborador possa compreender o porquê de tal recomendação (CASTRO et al., 2013).

Também foi observado que, no planejamento dos cardápios, a carne passou a ser retirada um dia antes, sendo levada a um refrigerador para que no dia seguinte seja limpa e cortada, sendo colocada para refrigerar até o dia seguinte para ser preparada e distribuída na refeição. Também foi observado que diminuiu o desperdício de alimentos, os cardápios atendiam a todos os requisitos, onde é servido uma proteína prato principal uma guarnição, saladas cruas e cozidas, o complemento arroz e feijão e uma fruta. Isto acontece devido a universidade contribuir com gêneros alimentícios para merenda escolar, como também adquire itens (frutas e legumes) da agricultura familiar recurso do FNDE/ PNAE (AQUISIÇÃO DE PRODUTOS DA AGRICULTURA FAMILIAR PARA O PROGRAMA NACIONAL DE ALIMENTAÇÃO ESCOLAR).

A Lei $n^{\circ} 11.947$, de 16 de junho de 2009, determina que no mínimo $30 \%$ do valor repassado a estados, municípios e Distrito Federal pelo Fundo Nacional de Desenvolvimento da Educação (FNDE) para o Programa Nacional de Alimentação Escolar (PNAE) deve ser utilizado na compra de gêneros alimentícios diretamente da agricultura familiar e do empreendedor familiar rural ou de suas organizações, priorizando-se os assentamentos da reforma agrária, as comunidades tradicionais indígenas e as comunidades quilombolas. A aquisição dos produtos da Agricultura Familiar poderá ser realizada por meio da Chamada Pública, dispensando-se, nesse caso, o procedimento licitatório (BRASIL, 2009).

Adicionalmente, foi observado uma atitude por parte da gestora, na qual as crianças recebiam instruções sobre a importância do consumo de frutas e hortaliças diariamente. A 
partir dessa ação, houve melhor aceitabilidade desses alimentos pelas crianças. De acordo com Paiva et al. (2012), a presença de um gestor na UAN de uma escola é fundamental para garantir a oferta de uma alimentação equilibrada e variada, além de contribuir para práticas educativas que visam aumentar o consumo de todos os grupos de alimentos oferecidos, em especial frutas, legumes e verduras.

Com relação aos adolescentes da turma do $9^{\circ}$ ano, foi criado um horário para ministrar atividades teóricas e práticas sobre segurança alimentar e alimentação saudável, incluindo preparo de receitas. Em si tratando de um público onde as escolhas alimentares são baseadas em doces, frituras, refrigerantes entre outros alimentos ricos em açúcares e gorduras que estão associados ao desenvolvimento de doenças crônicas não transmissíveis (MARRÓN-PONCE et al., 2019; LOUZADA et al., 2015), essa abordagem dentro da escola, fortalece o vínculo entre a escola e a comunidade.

Um fato que se destacou, foi a comunicação entre a gestora e os colaboradores, por exemplo, sempre que ia acontecer uma preparação nova, uma mudança na cozinha, a gestora comunicava se sempre com os colaboradores, isso só contribuiu para os colaboradores adquirirem mais confiança. Diante do exposto foi observado que os colaboradores passaram a ter mais compromisso com suas atividades, respeito uns com os outros, todas as vezes que um colaborador fosse faltar era levado ao conhecimento da gestora. Adicionalmente, foi observada melhor interação entre a equipe, criando um ambiente de trabalho satisfatório entre eles e a gestora.

Segundo Marchiori (2017), "a comunicação serve como “... uma ponte de significados que cria compreensão mútua e confiança, já que a compreensão leva a aceitação ou não da mensagem, e uma ação para quem recebe a mensagem".

Para Furnham (2001), "as pesquisas mostram que a escolha de canal de comunicação pode afetar em muito o nível de clareza ou ambiguidade da mensagem que está sendo transmitida. O canal oral (conversas pelo telefone ou reuniões pessoais, por exemplo) são preferíveis ao canal por escrito, com anotações e memorandos, quando as mensagens forem ambíguas (e exigirem bastante assistência para serem interpretadas) ao passo que o canal por escrito é preferível quando a mensagem é clara".

\section{Conclusões}

Podemos concluir que, a presença do gestor nessa UAN foi fundamental para instituir e padronizar técnica que visam obter qualidade ponto de vista higiênico-sanitário, desde o início da produção até o produto final, assim como para melhorar a interação entre a equipe. 


\section{Referências}

ABREU, E. S.; SPINELli, M. G. N.; PINTO, A. M. S. (Ed. 5). Gestão de Unidades de Alimentação e Nutrição: Um modo de fazer. São Paulo: Metha, 2013.

ALVAREZ, M. E. B. Gestão de qualidade, produção e operações. São Paulo: Atlas, (Ed. 3), 2019.

AMARAL, L. B.; CARNEVALE, R. M. S. Redução do desperdício de alimentos na produção de refeições hospitalares. Trabalho de Conclusão de Curso de especialização em Gestão Pública. Faculdade IBGEN - Instituto Brasileiro de Gestão De negócios, Porto Alegre, RS, 2008.

AVElATO, H.; ARAÚJO, E. M. G. Gestão, organização e condições de trabalho. V Congresso Nacional de Excelência em Gestão. Gestão do Conhecimento para a Sustentabilidade. 2009.

BITENCOURT, C. C. Gestão de Competências e Aprendizagem nas Organizações. São Leopoldo: UNISINOS, 2005.

BONI, V.; QUARESMA, S. J. Aprendendo a entrevistar: como fazer entrevistas em ciências sociais. Revista Eletrônica dos Pós-Graduandos em Sociologia Política da UFSC, v. 2, p. 68-80, 2005.

BRASIL. Ministério da Saúde. Resolução RDC no 216, de 15 de setembro de 2004. Aprova o regulamento técnico de boas práticas para serviços de alimentação. Disponível em: < http://portal.anvisa.gov.br/documents/33916/388704/RESOLU\%25C3\%2587\%25C3\%2583O RDC\%2BN\%2B216\%2BDE\%2B15\%2BDE\%2BSETEMBRO\%2BDE\%2B2004.pdf/237014 96-925d-4d4d-99aa-9d479b316c4b>. Acesso em: 05 de Jan. de 2018.

BRASIL. Resolução/CD/FNDE n. 38, de 16 de julho de 2009. Dispõe sobre o atendimento da alimentação escolar aos alunos da educação básica no Programa Nacional de Alimentação Escolar - PNAE. 2009. Disponível em: $<$ https://www.fnde.gov.br/index.php/acesso-a-informacao/institucional/legislacao/item/3341- 
resolu\%C3\%A7\%C3\%A3o-cd-fnde-n\%C2\%BA-38-de-16-de-julho-de-2009>. Acesso em: 05 de Jan. de 2018.

BRAVO, I. Gestão da qualidade em tempos de mudança. São Paulo: Alínea, (Ed. 3), 2010.

BOOG, M. C. F.; RODRIGUES, K. R. M.; SILVA, S. M. F. Situação profissional dos nutricionistas egressos da PUCCAMP 1. Áreas de atuação, estabilidade, abandono da profissão, desemprego. REVISTA DE NUTRIÇÃO DA PUCCAMP, V. 1, P. 139-152, 1988.

CASTRO, R. S. D.; VIEITES, R. L. Boas práticas de fabricação (BPF), análise de tomate e água em restaurantes da cidade de Botucatu - SP. Tese. Universidade Estadual Paulista. Júlio de Mesquita Filho. São Paulo: Botucatu, 2013.

CFN. CONSELHO FEDERAL DE NUTRICIONISTAS. Resolução $N^{\circ}$ 380, de 9 de Dezembro de 2005. Dispõe sobre a definição das áreas de atuação do nutricionista e suas atribuições, estabelece parâmetros numéricos de referência, por área de atuação. Brasília, DF. Disponível Em: < HTTP://WWW.CFN.ORG.BR/INICIAL/RESOLUCAO_380.PDF >. ACESSO EM 05 JAN. DE 2018.

COLARES, L. G. T.; FREITAS, C. M. Processo de trabalho, saúde e qualidade de vida no trabalho em uma unidade de alimentação e nutrição: Uma abordagem qualitativa. Tese. Escola Nacional De Saúde Pública, Rio De Janeiro, rj, 2005.

DE BRITO, K. M. F.; TORRES, C. C. Riscos à saúde dos trabalhadores em unidade de alimentação e nutrição de um hospital universitário. Dissertação. Universidade Federal do Rio Grande do Norte. Natal, RN, 2015.

FAYOL, H. Administração industrial e geral. São Paulo: ATLAS, 1994, P. 138.

FONSECA, M. T. Tecnologias gerenciais de restaurantes. São Paulo: SENAC, (ED. 7). 2018. 
FREITAS, P. F. P.; ODELIUS, C. C. Competências gerenciais: uma análise de classificações em estudos empíricos. Cad. EBAPE.BR, v. 16, 2018.

FURNHAM, A. Linguagem corporal no trabalho. (Tradução de Márcia da Cruz Nóboa Leme). São Paulo: Nobel, 2001.

JUSTEN, M. K.; CHILANTI, G. Absenteísmo em uma unidade de alimentação e nutrição de grande porte em Caxias do Sul-RS. (Trabalho de Conclusão de Curso de Graduação, de Bacharelado em Nutrição). Universidade de Caxias do Sul, Canela, RS, 2017.

LOUZADA, M. L. C.; et al. Consumption of ultra-processed foods and obesity in Brazilian adolescents and adults. Prev Med. v.81, p. 9-15, 2015.

MANZALLI, P. V. Manual para serviços de alimentação. São Paulo, Metha, (Ed. 2), 2010.

MARCHIORI, M. Cultura e comunicação organizacional. São Caetano do Sul: Difusão, (Ed. 2), 2017.

MAXIMIANO, A. C. A Introdução à Administração. São Paulo: Atlas, (Ed. 8), 2011.

MARRÓN-PONCE, J. A., et al. Associations between Consumption of Ultra-Processed Foods and Intake of Nutrients Related to Chronic Non-Communicable Diseases in Mexico. J Acad Nutr Diet. v.119, p. 1852-1865, 2019.

MEZOMO, I. B. Os Serviços de Alimentação: Planejamento e Administração. São Paulo: Manole, (Ed. 6), 2014.

MINAYO, M. C. S. O desafio do conhecimento: pesquisa qualitativa em saúde. São Paulo: Hucitec Abrasco, (Ed. 14), 2014. p. 393.

MOREIRA, T. R. B. M.; DAL FABBRO, A. L. Condições sanitárias e documentação de serviços de alimentação de creches públicas e privadas. (Dissertação). Universidade de São Paulo, Ribeirão Preto, SP. 2018. 
NOBREGA, A. B. N.; et al. Competências gerenciais do nutricionista gestor de unidades de alimentação terceirizada. RapUnP. 2012.

OLIVEIRA, S. P. F.; FERREIRA, A. M. M.; TEIXEIRA, G. M. Qualidade de vida no trabalho de manipuladores de um serviço de nutrição hospitalar em Maceió - AL. Revista Eletrônica Acervo Saúde, v.50, p. e3498, 2020.

PAIVA, P. Y. U.; et al. A importância do nutricionista na qualidade de refeições escolares: estudo comparativo de cardápios de escolas particulares de ensino infantil no município de São Paulo. Revista UNIVAP, São José dos Campos-SP, v. 18, dez.2012.

PALADINI, Edison Pacheco. Gestão da qualidade: teoria e prática. São Paulo: Atlas, (Ed. 3), 2012.

ROSA, P. T.; GUELBERT, T. F. Implantação do manual de boas práticas de manipulação em cozinha pedagógica de uma instituição de ensino na cidade de Campo Mourão - PR. (Trabalho de Conclusão de Curso de Graduação, do Curso Superior de Tecnologia de Alimentos), Universidade Tecnológica Federal do Paraná. Campo Mourão, PR, 2015.

SANTOS, M.; BUCCIOLI, P. T. Motivação e liderança em Unidades de Alimentação e Nutrição. Revista Ciências Nutricionais Online, v.3, p.39-45, 2019.

SARTOR, J.; ALVES, M. K. Percepção do perfil de liderança do nutricionista gestor em unidades de alimentação e nutrição. Revista Saúde e Desenvolvimento Humano. v. 7, 2019.

SILVA, S. M. R; BRASIL, D. S. B. Importância da utilização das ferramentas de gestão da qualidade para a produção de alimentos seguros - análise de uma unidade de alimentação e nutrição (UAN) na cidade de Belém - PA. (Dissertação), Universidade Lusófona de Humanidades e Tecnologias Departamento de Economia e Gestão, Lisboa, 2012.

SLACK, N. Administração da produção. São Paulo: Atlas, (Ed. 8), 2018.

TEIXEIRA, S. et al. Administração aplicada a Unidades de Alimentação e Nutrição. São Paulo: Atheneu, (Ed. 1), 2010. 